\title{
Prognostic significance of the neutrophil-to-lymphocyte ratio and platelet-to-lymphocyte ratio for advanced non-small cell lung cancer patients with high PD-L1 tumor expression receiving pembrolizumab
}

\author{
Doran Ksienski ${ }^{1,2}$, Elaine S. Wai ${ }^{1,2}$, Deepu Alex ${ }^{3}$, Nicole S. Croteau ${ }^{4}$, Ashley T. Freeman ${ }^{1,2}$, Angela Chan ${ }^{2,5}$, \\ Tiffany Patterson ${ }^{1}$, Melissa Clarkson ${ }^{1}$, Leathia Fiorino ${ }^{1,2}, \mathrm{Zia}_{\text {Poonja }}{ }^{1,2}$, David Fenton ${ }^{1,2}$, Sarah Irons ${ }^{1}$, \\ Mary Lesperance ${ }^{6}$
}

${ }^{1}$ BC Cancer-Victoria, Victoria, BC, Canada; ${ }^{2}$ Department of Medical Oncology, University of British Columbia, Vancouver, BC, Canada; ${ }^{3}$ BC CancerVancouver, Vancouver, BC, Canada; ${ }^{4}$ Department of Anesthesiology, Pharmacology, \& Therapeutics, University of British Columbia, Vancouver, BC, Canada; ${ }^{5}$ BC Cancer-Surrey, Surrey, BC, Canada; ${ }^{6}$ Department of Mathematics and Statistics, University of Victoria, Victoria, BC, Canada

Contributions: (I) Conception and design: All authors; (II) Administrative support: S Irons, D Ksienski, NS Croteau; (III) Provision of study materials or patients: D Ksienski; (IV) Collection and assembly of data: D Ksienski, NS Croteau, M Clarkson, T Patterson, AT Freeman, M Lesperance; (V) Data analysis and interpretation: D Ksienski, ES Wai, NS Croteau, M Lesperance; (VI) Manuscript writing: All authors; (VII) Final approval of manuscript: All authors.

Correspondence to: Doran Ksienski, MD, FRCPC, MPH. BC Cancer-Victoria, 2410 Lee Avenue V8R 6V5, Victoria, BC, Canada.

Email: dksienski@bccancer.bc.ca.

Background: We investigated the association of peripheral blood inflammatory markers with overall survival (OS) in pembrolizumab treated advanced non-small cell lung cancer (aNSCLC) patients with programmed death ligand 1 (PD-L1) expression $\geq 50 \%$. Clinical risk factors for development of immunerelated adverse events (irAE) were also explored.

Methods: aNSCLC patients with high PD-L1 expression receiving pembrolizumab monotherapy outside of clinical trials were identified retrospectively. All patients were treated at one of six British Columbia Cancer clinics between August 2017 and June 2019. Patients were dichotomized using baseline neutrophilto-lymphocyte ratio (NLR, $</ \geq 6.4$ ) and platelet-to-lymphocyte ratio (PLR, $</ \geq 441.8$ ). Factors associated with OS were assessed with Cox proportional hazard models. Logistic regression models were utilized in landmark analysis of risk factors for irAE.

Results: Among 220 patients, median age was 70.0 years, 55.0\% were female, 40.5\% had baseline Eastern Cooperative Oncology Group performance status (ECOG PS) 2/3, and 95.5\% received frontline pembrolizumab. Median OS for the cohort was 11.8 months (95\% CI: 8.7-15.4). On multivariable analysis, baseline NLR $\geq 6.4$ [hazard ratio (HR): 2.31, 95\% confidence interval (CI): 1.46-3.64, $\mathrm{P}<0.001$ ], baseline PLR $\geq 441.8$ (HR: 2.03, 95\% CI 1.22-3.37, P=0.006), and pre-treatment ECOG PS 2/3 (HR: 2.19, 95\% CI: $1.48-3.26, \mathrm{P}<0.001)$ were associated with worse OS. The incidence of any grade irAE and irAE grade $\geq 3$ were $40.5 \%$ and $12.3 \%$, respectively. ECOG PS $2 / 3$ (vs. 0/1) patients were at 3.76-fold higher risk of developing an irAE by the 8-month landmark ( $\mathrm{P}=0.002)$.

Conclusions: High NLR and PLR were associated with shorter OS in a cohort of patients receiving largely frontline pembrolizumab for aNSCLC in routine practice. ECOG PS 2/3 was associated with higher risk of developing an irAE. Given that NLR and PLR values are easily obtainable, prospective studies are warranted to confirm their prognostic significance in this patient population and explore a predictive utility.

Keywords: Prognostic; biomarker; pembrolizumab; adverse events 
Submitted Apr 08, 2020. Accepted for publication Sep 19, 2020.

doi: $10.21037 /$ tlcr-20-541

View this article at: http://dx.doi.org/10.21037/tlcr-20-541

\section{Introduction}

Treatment with the programmed cell death 1 (PD-1) antibody pembrolizumab for advanced non-small cell lung cancer (aNSCLC), dramatically improves clinical outcomes for a subset of patients (1-4). About $23-28 \%$ of aNSCLC patients have a programmed death ligand 1 (PD-L1) tumor proportion score (TPS) $\geq 50 \%(1,3)$. Tumoral PD-L1 can bind to PD-1 receptors on activated T cells, resulting in evasion of immune response. Randomized controlled trials have demonstrated improved overall survival (OS) amongst treatment naïve aNSCLC patients with a PDL1 TPS $\geq 50 \%$ [but without an epidermal growth factor receptor (EGFR) mutation or anaplastic lymphoma kinase (ALK) rearrangement] who receive pembrolizumab compared to platinum doublet chemotherapy $(3,4)$. As a second line therapy after progression on platinum chemotherapy, survival outcomes are superior in PD-L1 positive aNSCLC patients treated with pembrolizumab versus docetaxel chemotherapy (2). However, the minority of patients in these clinical trials responded to immunotherapy and identification of predictive and prognostic biomarkers remains an unmet need (5). Tumor mutation burden is a potential predictive marker however the test is expensive and likely difficult to implement in routine clinical practice (6).

Cancer-related inflammation has a role in tumorigenesis, disease progression, and patient prognosis (7). Inflammatory biomarkers such as the neutrophil-to-lymphocyte ratio (NLR) and platelet-to-lymphocyte ratio (PLR) are prognostic indicators in solid tumors $(8,9)$. These bloodbased biomarkers are easily obtainable as part of routine tests. In aNSCLC patients receiving predominately nivolumab, elevated NLR (10-16) and PLR (16-18) have been correlated with worse OS. However, the prognostic significance of NLR and PLR in aNSCLC patients with high PD-L1 expression receiving pembrolizumab is unclear. Other candidate blood-based biomarker ratios associated with clinical outcome include the derived NLR (either alone or as part of the lung immune prognostic index) and monocyte to lymphocyte ratio $(13,19)$.

T-cell activation can cause a unique spectrum of sideeffects termed immune-related adverse events (irAE), potentially requiring prolonged use of steroids and immune modulating agents (20). As individuals treated in everyday clinical practice are older and have worse Eastern Cooperative Group Oncology performance status (ECOG PS) than those enrolled into clinical trials, it is important to describe the incidence and severity of irAE in a real world setting $(21,22)$. In addition, identification of clinical risk factors for immune toxicity are needed to assist in risk stratification and monitoring strategies $(23,24)$.

In this retrospective series of aNSCLC patients with a PD-L1 TPS $\geq 50 \%$ receiving pembrolizumab in routine clinical practice, our primary goal was to evaluate the association of baseline NLR and PLR with clinical outcomes. We hypothesized that elevated pre-treatment NLR and PLR would be associated with poorer OS. As a secondary objective, clinical risk factors associated with the development of irAE were investigated. We present the following article in accordance with the Reporting Recommendations for Tumor Marker Prognostic Studies (REMARK) reporting checklist (25) (available at http:// dx.doi.org/10.21037/tlcr-20-541).

\section{Methods}

\section{Patients and setting}

The study was conducted in accordance with the Declaration of Helsinki (as revised in 2013). The study was approved by the institutional ethics board of University of British Columbia Research Ethics Board (H19-01328) and individual consent for this retrospective analysis was waived. Cases were selected from aNSCLC patients (stage IV, 7th edition UICC TNM classification or recurrent nonresectable disease not amenable to curative intent radiotherapy) treated at British Columbia (BC) Cancer. The BC Cancer Provincial Pharmacy Database contains records of all systemic therapy delivered. After approval of the study protocol from the University of British Columbia Research Ethics Board, the pharmacy database was searched for aNSCLC patients treated with pembrolizumab between August 2017 and June 2019. Inclusion criteria for the current study were: use of pembrolizumab (in any line) for treatment of aNSCLC and a PD-L1 TPS $\geq 50 \%$. 
Patients receiving pembrolizumab as part of a clinical trial or concurrently with chemotherapy were excluded. Pembrolizumab was administered at a dose of $2 \mathrm{mg} / \mathrm{kg}$ intravenously every 3 weeks until progression, intolerance, or for 35 cycles (whichever came first) as per institutional treatment protocols (26).

\section{Tumor specimen characteristics}

Tumor samples were obtained at the time of biopsy and fixed in $10 \%$ buffered formalin. Subsequently, the samples were dehydrated in ethanol and paraffin embedded following standard procedures. Four micron tissue sections were cut and mounted onto histologic glass slides for examination with hematoxylin and eosin staining and PD-L1 immunohistochemistry (IHC). Positive and negative controls for IHC were run as per manufacturer specifications (27). Formalin fixed paraffin embedded (FFPE) blocks are stored at room temperature for repeat or additional studies as clinically indicated.

\section{Assay methods}

PD-L1 IHC and assessment is performed using FDAapproved 22C3 pharmDx (27) with Autostainer Link 48 as per manufacturer specifications. PD-L1 IHC 22C3 pharmDx is a quantitative immunohistochemical assay using mouse anti-PD-L1, Clone 22C3, for the detection of PD-L1 protein in FFPE NSCLC tissue. Unstained slide sections are initially incubated with the primary monoclonal antibody to PD-L1 followed by incubation with a linker antibody specific to the host species of the primary antibody. Subsequently, these slides are incubated with a ready to use visualization reagent consisting of secondary antibodies and horseradish peroxidase coupled to a dextran polymer backbone. Enzymatic conversion of this chromogen results in a visible reaction at the site of antigen/PD-L1 presence. The color of the chromogenic reaction is modified by a chromogen enhancement reagent. The slide is then counterstained and cover-slipped. PD-L1 protein expression is calculated by using TPS which is the percentage of viable tumor cells showing partial or complete membrane staining. The PD-L1 scores are reported by a board-certified pathologist as percentages belonging to one of three categories: less than $1 \%, 1 \%$ to $49 \%$ and, greater than or equal to $50 \%$. The PD-L1 scores were performed and reported separately for clinical use with no knowledge of the study's endpoints.

\section{Data collection}

Clinical data was extracted retrospectively from chart review from initial Medical Oncology consultation until November 2019. Furthermore, clinical notes were analyzed from date of first pembrolizumab treatment to last follow-up to document the incidence, severity, and treatment of irAE. Identification of irAE was based on the treating healthcare practitioner's assessment while grade was assigned by the abstractor utilizing Common Terminology Criteria for Adverse Events, version 4 (28).

\section{Clinical endpoints}

For the primary outcome, OS was defined as the time from first pembrolizumab treatment until date of death. Progression free survival (PFS) was measured from pembrolizumab start to date of physician determined progression or death. Patients not experiencing an event of interest were censored at last follow-up. Secondary aims were to explore the association of baseline clinical factors and the development of irAE (any grade).

\section{Statistical analysis}

Data are presented as percentages and counts for categorical variables and by the mean and interquartile ranges (IQRs) for continuous variables. Association between baseline NLR and PLR and patient characteristics were assessed using Pearson's Chi-squared, Fisher's exact, and Kruskal-Wallis rank sum tests as appropriate. Median follow-up times were computed (I) as the median of all survival times (ignoring censoring) and (II) by the reverse Kaplan-Meier method which provides an estimate of the potential follow-up (29). Survival curves were plotted with the Kaplan-Meier method and baseline factors explored for potential prognostic utility using the log-rank test.

Univariable and multivariable Cox proportional hazards models were used to generate point estimates of hazard ratios (HRs) and the corresponding 95\% confidence interval (CI) for candidate variables and OS. Univariable and multivariable logistic regression models were used to determine the odds of experiencing an irAE within 6 and 8 months of pembrolizumab initiation. Included in this analysis were patients who experience an irAE between pembrolizumab initiation and landmark time, plus patients not experiencing irAE during this period but who were alive at the landmark. Six- and 8-month landmarks chosen as 
most irAE would likely have developed by these time points. For multivariable analysis where there were fewer than 10 events per variable, a reduced model was fit via backwards stepwise regression. Candidate variables considered for inclusion in models of OS were: age at aNSCLC diagnosis, gender, baseline ECOG PS, Charlson comorbidity index (CCI), presentation with aNSCLC, presence of baseline brain or liver metastases, smoking status, histology, oncogenic alteration (EGFR mutation or ALK rearrangement), prior palliative chemotherapy, baseline body mass index (BMI), baseline NLR, and baseline PLR. Similar candidate variables were considered for inclusion in irAE models with the exception of CCI, smoking status, presentation with aNSCLC, line of pembrolizumab treatment, presence of oncogenic alteration, and BMI.

NLR (defined as absolute neutrophil count/platelet count) and PLR (defined as platelet count/absolute lymphocyte count) ratios were calculated based on blood work prior to cycle 1 of pembrolizumab. Optimal cut-off points for baseline NLR and PLR were identified using an outcome-oriented method to maximize differences in OS in the groups above and below the cut-off as suggested by Delgado et al. (30). Two groups of patients with high ( $\geq$ cut-off) and low (<cut-off) NLR, and separately for PLR, were delineated using maximally selected rank statistics as implemented in the $\mathrm{R}$ 'maxstat' package.

In an exploratory analysis to determine whether differences in OS were associated with variation in baseline neutrophil, lymphocytes, or platelet values, the cohort was divided into quartiles for each blood cell count (10). Kaplan-Meier survival curves for quartiles were generated and compared using log-rank tests. Post-hoc pairwise comparisons were performed and $\mathrm{P}$ values adjusted for multiple comparisons by the Bonferonni method $\left(\mathrm{P}_{\text {adi }}\right)$.

All $\mathrm{P}$ values were based on two sided hypotheses tests and those less than 0.05 were considered statistically significant. Statistical analyses were performed using $\mathrm{R}$ version 3.6.1 and $\mathrm{R}$ packages 'survival' version 2.44, 'survminer' version 0.4.6, 'maxstat' 0.7-25, and 'tableone' version 0.10.0 (31-36).

\section{Results}

\section{Patient characteristics}

A total of 279 aNSCLC patients treated with pembrolizumab for aNSCLC in the studied time period were identified; 59 patients had a PD-L1 TPS 1-49\% and were excluded from analysis. Baseline characteristics of the 220 patients meeting eligibility criteria are described in Table 1. Sixty-eight patients $(30.9 \%)$ were $\geq 75$ years old at diagnosis of aNSCLC, 121 patients (55.0\%) were female, and 89 patients (40.5\%) had ECOG PS $2 / 3$ at pembrolizumab initiation. Most had non-squamous histology [175 patients (79.5\%)] and nearly all lacked an oncogenic driver mutation [217 patients (98.6\%)]. The $95.5 \%$ of individuals received frontline pembrolizumab while the remainder had progressed on a platinum doublet.

\section{Baseline NLR and PLR}

Pre-treatment hematologic values were available for all patients in the studied cohort. At baseline, the median NLR was 5.5 (IQR: 3.3-8.9) and median PLR was 262.1 (IQR: 175.6-411.9). An optimal cut-off for NLR of 6.4 stratified patients into high NLR [ $\geq 6.4, n=92,41.8 \%$ of cohort] and low NLR $(<6.4, n=128,58.2 \%)$. For PLR, the threshold value was 441.8 and stratified patients into high $\operatorname{PLR}(\geq 441.8, \mathrm{n}=50,22.7 \%)$ and low PLR $(<441.8, \mathrm{n}=170$, $77.3 \%)$.

The relation of NLR and PLR with baseline clinicopathologic characteristics are shown in Table 2. Patients with high ( $v s$. low) baseline PLR were more likely to have a high baseline NLR $(\mathrm{P}<0.001)$ and $\mathrm{BMI}<25 \mathrm{~kg} / \mathrm{m}^{2}$ $(\mathrm{P}<0.001)$. High baseline NLR was significantly associated with younger age at aNSCLC diagnosis $(\mathrm{P}=0.002)$, ECOG PS $2 / 3(\mathrm{P}=0.004)$, high baseline PLR $(\mathrm{P}<0.001)$, and a BMI $<25 \mathrm{~kg} / \mathrm{m}^{2}(\mathrm{P}=0.002)$ (Table 2). There was a strong positive correlation between baseline continuous measurements of NLR and PLR (Spearman's rho $=0.73, \mathrm{P}<0.001$ ).

\section{Survival analysis}

Calculated simply, median follow-up time was 9.2 (range, 0.03-25) months and 16.1 months by the reverse KaplanMeier method. At last follow-up, 125 (57.0\%) patients had died and $52(24 \%)$ were still receiving pembrolizumab. The median OS and PFS for the whole cohort was 11.8 months (95\% CI: 8.7-15.4) and 6.1 months (95\% CI: 5.0-8.3) respectively.

\section{Association of baseline NLR and PLR with clinical outcomes}

Patients with a low (vs. high) baseline NLR were observed to have a longer OS (median 18.9 vs. 5.4 months, logrank $\mathrm{P}<0.001$ ) and PFS (median 10.0 vs. 3.5 months, log- 
Table 1 Patient characteristics at baseline $(n=220)$

\begin{tabular}{|c|c|}
\hline Characteristic & $\mathrm{N}(\%)$ \\
\hline Age at aNSCLC diagnosis, median (IQR) & $70.0(62.8-76.0)$ years \\
\hline \multicolumn{2}{|l|}{ Gender } \\
\hline Male & $99(45.0)$ \\
\hline Female & $121(55.0)$ \\
\hline \multicolumn{2}{|l|}{ ECOG PS } \\
\hline $0 / 1$ & $131(59.5)$ \\
\hline $2 / 3$ & $89(40.5)$ \\
\hline CCl, median (IQR) & $1.0(1.0-3.0)$ \\
\hline \multicolumn{2}{|l|}{ Smoking status } \\
\hline Current & $79(35.9)$ \\
\hline Former & $122(55.5)$ \\
\hline Never & $19(8.6)$ \\
\hline \multicolumn{2}{|l|}{ Histology } \\
\hline Non-squamous & $175(79.5)$ \\
\hline Squamous & $45(20.5)$ \\
\hline aNSCLC at presentation & $181(82.3)$ \\
\hline EGFR mutation & $3(1.4)$ \\
\hline ALK rearrangement & $0(0.0)$ \\
\hline Liver metastases & $18(8.2)$ \\
\hline Brain metastases & $30(13.6)$ \\
\hline $\begin{array}{l}\text { Radiotherapy anytime during } \\
\text { pembrolizumab treatment }\end{array}$ & $36(16.4)$ \\
\hline \multicolumn{2}{|l|}{ Prior line of palliative systemic therapy } \\
\hline 0 & $210(95.5)$ \\
\hline 1 & $10(4.5)$ \\
\hline \multicolumn{2}{|l|}{ BMI } \\
\hline$\geq 25 \mathrm{~kg} / \mathrm{m}^{2}$ & $96(43.6)$ \\
\hline$<25 \mathrm{~kg} / \mathrm{m}^{2}$ & $112(50.9)$ \\
\hline Unknown & $12(5.5)$ \\
\hline Baseline NLR, median (IQR) & $5.5(3.3-8.9)$ \\
\hline Baseline PLR, median (IQR) & $262.1(175.6-411.9)$ \\
\hline
\end{tabular}

aNSCLC, advanced non-small cell lung cancer; IQR, interquartile range; ECOG PS, Eastern Cooperative Oncology Group performance status; $\mathrm{CCl}$, Charlson comorbidity index; EGFR, epidermal growth factor receptor; ALK, anaplastic lymphoma kinase; BMI, body mass index; NLR, neutrophil-to-lymphocyte ratio; PLR, platelet-to-lymphocyte ratio; rank $\mathrm{P}<0.001$ ) (Figure 1). Similarly, a low baseline PLR was associated with longer OS (median 15.4 vs. 4.0 months, logrank $\mathrm{P}<0.001$ ) and PFS (median 6.7 vs. 2.9 months, log-rank $\mathrm{P}<0.001)$ (Figure 1). Additional cut-off points for baseline $\operatorname{NLR}(<3$ vs. $\geq 3$ and $<5$ vs. $\geq 5)$ and PLR $(<180 v s . \geq 180)$ were assessed and can be found in Figure S1.

On univariable analysis the following covariates in the studied cohort were associated with worse OS: baseline NLR $\geq 6.4$ (HR: 2.59, 95\% CI: 1.82-3.70, P<0.001), baseline PLR $\geq 441.8$ (HR: 2.55, 95\% CI: 1.74-3.72, $\mathrm{P}<0.001$ ), ECOG PS 2/3 (HR: 2.31, 95\% CI: $1.62-3.30$, $\mathrm{P}<0.001$ ), and squamous histology (HR: $1.58,95 \% \mathrm{CI}$ : 1.05-2.36, $\mathrm{P}=0.03$ ) (Table 3). On multivariable analysis, baseline NLR $\geq 6.4$ (HR: 2.31, 95\% CI: 1.46-3.64), baseline PLR $\geq 441.8$ (HR: 2.03, 95\% CI: 1.22-3.37, $\mathrm{P}=0.006$ ), and ECOG PS 2/3 (HR: 2.19, 95\% CI: 1.48-3.26, P<0.001) were associated with poorer OS. The presence of liver metastasis at start of pembrolizumab treatment trended towards shorter OS (HR: 1.86, 95\% CI: 0.96-3.61, $\mathrm{P}=0.07$ ).

\section{Exploratory analysis of baseline hematologic values}

To assess whether differences in OS were attributable to variation in baseline neutrophil, lymphocytes, or platelet values, the cohort was divided into quartiles for each blood cell count (10). Neutrophil quartiles were 1.30-4.40 ( $\mathrm{n}=50)$, 4.40-6.10 ( $\mathrm{n}=57), 6.10-8.15(\mathrm{n}=58)$, and 8.15-46.2 $(\mathrm{n}=55)$. Lymphocyte quartiles were $0.20-0.70(\mathrm{n}=34), 0.70-1.15$ $(\mathrm{n}=76), 1.15-1.60(\mathrm{n}=52)$, and 1.60-4.90 $(\mathrm{n}=58)$. Platelet quartiles were 31.0-230.5 ( $\mathrm{n}=55), 230.5-306.5(\mathrm{n}=55)$, 306.5-383.5 ( $\mathrm{n}=55)$, and 383.5-937.0 $(\mathrm{n}=55)$. As shown in Figure 2, patients in the highest (fourth) quartile for neutrophils had poorer survival compared to all other quartiles (all pairwise log-rank $\mathrm{P}_{\text {adj }} \leq 0.03$ ); when analyzing survival outcomes by baseline lymphocyte count, those in the lowest (first) quartile demonstrated the shortest survival to all other quartiles (all pairwise log-rank $\mathrm{P}_{\text {adj }} \leq 0.001$ ). Regarding baseline platelet levels, patients in the highest (fourth) quartile had shorter OS compared to those in the lowest quartile $\left(\mathrm{P}_{\text {adj }}=0.04\right)$.

\section{Risk factors for irAE}

In the entire cohort of aNSCLC patients receiving pembrolizumab, 127 separate irAE were observed. 89 patients $(40.5 \%$ of cohort) experienced an irAE; the 
Table 2 Patient characteristics stratified by baseline NLR and PLR status

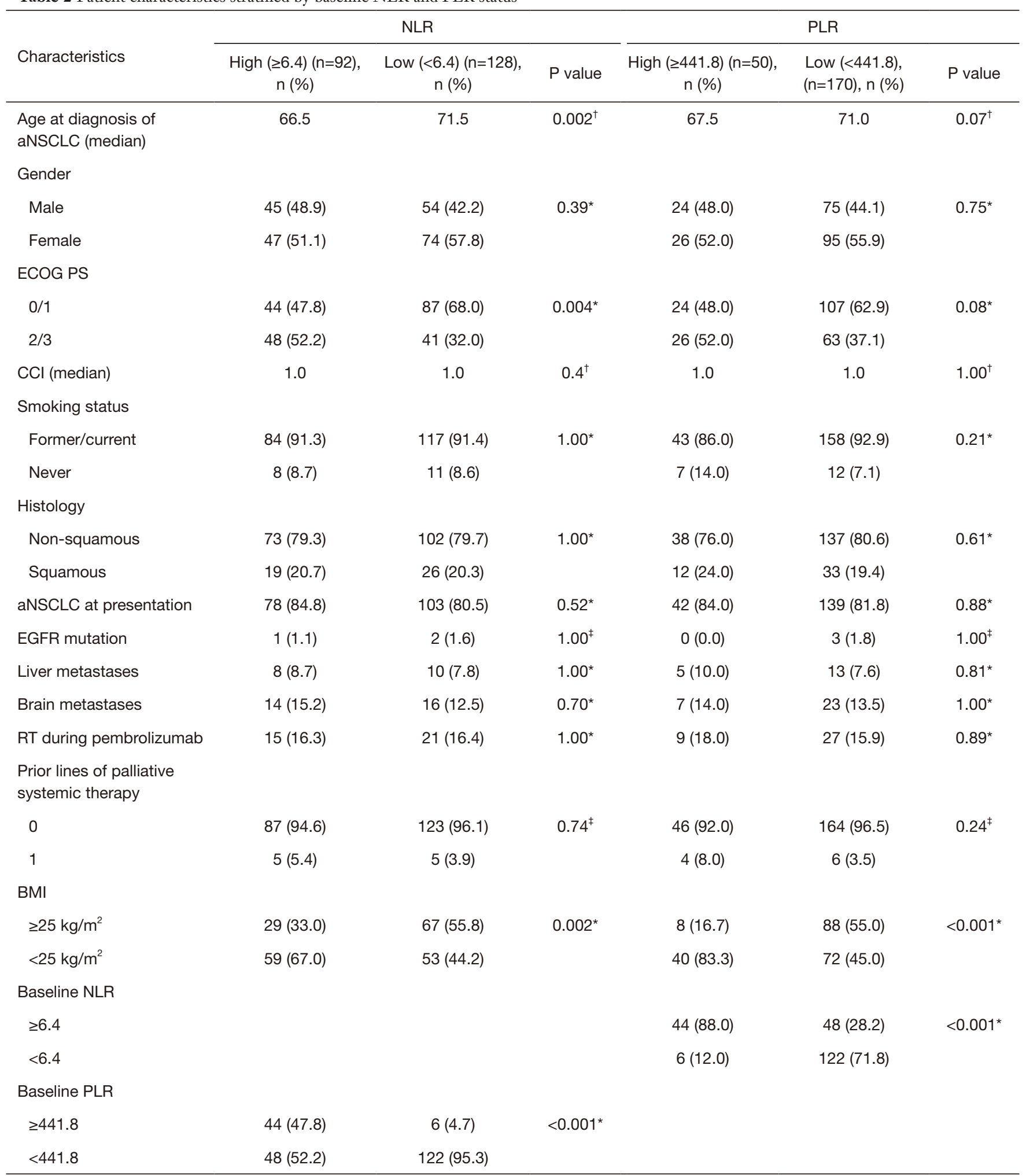

*, P value for Chi-squared test; ${ }^{\dagger}$, P value for Kruskal-Wallis rank sum test; ${ }^{\ddagger}, \mathrm{P}$ value for Fisher's exact test. NLR, neutrophil-to-lymphocyte ratio; PLR, platelet-to-lymphocyte ratio; aNSCLC, advanced non-small cell lung cancer; ECOG PS, Eastern Cooperative Oncology Group performance status; CCI, Charlson comorbidity index; EGFR, epidermal growth factor receptor; RT, radiotherapy; BMI, body mass index. 


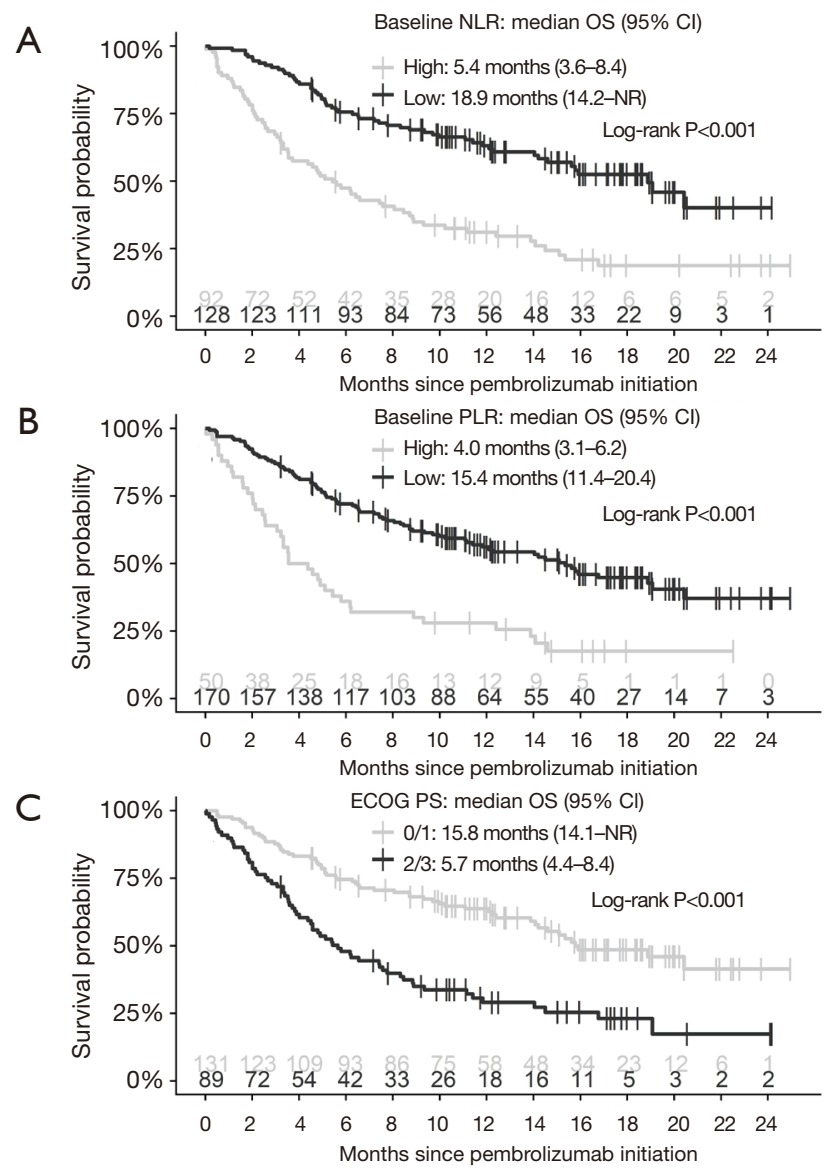

Figure 1 Kaplan-Meier curves of OS according to baseline (A) NLR (high $\geq 6.4$, low <6.4), (B) PLR (high $\geq 441.8$, low <441.8), and (C) ECOG PS (0/1 vs. 2/3). OS, overall survival; NLR, neutrophil-to-lymphocyte ratio; PLR, platelet-to-lymphocyte ratio; ECOG PS, Eastern Cooperative Oncology Group performance status; CI, confidence interval; NR, not reached.

incidence irAE $\geq$ grade 3 was $12.3 \%$ (Table 4 ). One patient died from pneumonitis attributed to pembrolizumab therapy. The most commonly observed irAE were thyroid changes (10.5\%), colitis (9.5\%), and dermatitis (9.1\%). $7.7 \%$ of the cohort required a treatment delay due to immunetoxicity while $16.4 \%$ needed treatment discontinuation due to irAE. Amongst the 89 patients who experienced an irAE, 41 patients $(46.1 \%)$ required steroids and none were treated with immune modulating agents.

Univariable and multivariable logistic regression analysis was performed to assess factors predicting development of irAE (any grade) within 6 and 8 months of starting pembrolizumab. At the 6-month landmark, 147 patients were identified with 66 patients experiencing an irAE while 81 patients did not. On univariable analysis, ECOG PS 2/3 [odds ratio (OR): $1.98,95 \% \mathrm{CI}$ : $1.00-3.95, \mathrm{P}=0.051$ ] was associated with increased risk of irAE while baseline high PLR (OR: 2.09, 95\% CI: 0.88-5.16, $\mathrm{P}=0.10$ ) was a weaker risk factor (Table S1). Multivariable logistic regression of irAE manifestation within 6 months of pembrolizumab initiation demonstrated that ECOG PS 2/3 (OR: $1.88,95 \%$ CI: $0.92-3.92, \mathrm{P}=0.09$ ) was the only studied factor marginally associated with risk of irAE. For 8-month landmark analysis, 137 patients were identified with 75 patients experiencing an irAE while 62 did not. Multivariable logistic regression showed that poor ECOG PS (OR: 3.76, 95\% CI: 1.69-8.88, $\mathrm{P}=0.002$ ) significantly increased odds of irAE within 8 months of initiating pembrolizumab (Table S2).

\section{Discussion}

We evaluated the prognostic significance of NLR and PLR in a large cohort of aNSCLC patients with PD-L1 TPS 
Table 3 Univariable and multivariable Cox regression analysis of OS with baseline NLR and PLR ( $\mathrm{n}=220$ patients)

\begin{tabular}{|c|c|c|c|c|}
\hline Variables & \multicolumn{2}{|c|}{ Univariable } & \multicolumn{2}{|c|}{ Multivariable } \\
\hline Age ( $\geq 75$ vs. $<75)$ & $0.77(0.52-1.15)$ & 0.20 & $1.22(0.78-1.91)$ & 0.38 \\
\hline Gender (male vs. female) & $1.19(0.84-1.69)$ & 0.33 & $1.19(0.80-1.77)$ & 0.38 \\
\hline ECOG PS (2/3 vs. 0/1) & $2.31(1.62-3.30)$ & $<0.001$ & $2.19(1.48-3.26)$ & $<0.001$ \\
\hline Smoking status (never vs. current/former) & $1.28(0.71-2.33)$ & 0.41 & $1.22(0.65-2.32)$ & 0.54 \\
\hline EGFR/ALK mutation vs. none & $0.49(0.07-3.49)$ & 0.47 & & \\
\hline Liver metastases (present vs. none) & $1.65(0.93-2.94)$ & 0.09 & $1.86(0.96-3.61)$ & 0.07 \\
\hline Brain metastases (present vs. none) & $1.03(0.61-1.74)$ & 0.91 & $1.02(0.57-1.84)$ & 0.95 \\
\hline Baseline NLR ( $\geq 6.4$ vs. <6.4) & $2.59(1.82-3.70)$ & $<0.001$ & $2.31(1.46-3.64)$ & $<0.001$ \\
\hline Baseline PLR ( $\geq 441.8$ vs. <441.8) & $2.55(1.74-3.72)$ & $<0.001$ & $2.03(1.22-3.37)$ & 0.006 \\
\hline BMI ( $\geq 25$ vs. $<25$ kg/m²) & $0.88(0.61-1.26)$ & 0.49 & $1.26(0.83-1.92)$ & 0.29 \\
\hline Prior lines of palliative chemo ( 1 vs. 0$)$ & $1.04(0.42-2.54)$ & 0.94 & & \\
\hline
\end{tabular}

There are too few patients with EGFR/ALK mutations and second line pembrolizumab recipients to include in multivariable analysis. OS, overall survival; NLR, neutrophil-to-lymphocyte ratio; PLR, platelet-to-lymphocyte ratio; HR, hazard ratio; Cl, confidence interval; ECOG PS, Eastern Cooperative Oncology Group performance status; CCI, Charlson comorbidity index; EGFR, epidermal growth factor receptor; ALK, anaplastic lymphoma kinase; aNSCLC, advanced non-small cell lung cancer; BMI, body mass index.

$\geq 50 \%$ receiving predominately frontline pembrolizumab in routine clinical practice. Consistent with our initial hypothesis, high baseline NLR and PLR were associated with shorter OS on multivariable analysis. Furthermore ECOG PS 2/3 (vs. 0/1) at treatment initiation was associated with both a 2.19-fold increased risk of death and 3.76-fold greater risk of developing an irAE within 8 months of starting immunotherapy.

Baseline NLR and PLR have been associated with poor survival in aNSCLC patients receiving nivolumab in $\geq$ second line setting $(10,11)$. However specific cut-off values in published literature vary likely due to differences in patient baseline characteristics (age, gender, ethnicity, etc.), prior treatments (radiation or chemotherapy) and statistical methods $(13,15)$. For example, the optimal NLR threshold has been defined as $\geq 5.9(13), \geq 5(5,16), \geq 4$ (12), and $\geq 2.8$ (14). Similarly, cut-offs for PLR have been defined as $\geq 169$ (16), $\geq 164$ (17), and $\geq 180$ (18). Diem et al. categorized inflammatory blood markers into tertiles (NLR: <3.6, 3.6-6.5, and >6.5; PLR: $<193$, 193-328, and >328) (11). Importantly, PD-L1 expression was unknown in a large proportion of patients in the previously mentioned studies.

The current analysis, to the best of our knowledge, represents the largest study exploring the prognostic significance of NLR and PLR in pembrolizumab treated aNSCLC patients with PD-L1 TPS $\geq 50 \%$. In a cohort of 51 aNSCLC patients with high PD-L1 TPS receiving pembrolizumab, Hasegawa et al. observed baseline NLR $\geq 4.56$ to be associated with poorer OS (37). Our cut-off of $\geq 6.4$ for NLR and $\geq 441.8$ for PLR were determined using an outcome-oriented method that identified a value with the most significant relation to OS based on maximally selected rank statistics (35). On multivariable analysis of the studied cohort, we observed baseline NLR $\geq 6.4$ and PLR $\geq 441.8$ to be significantly and independently correlated with OS. Additional analyses using cut-offs from prior studies of NLR ( $\geq 3$ and $\geq 5)(10,15)$ and PLR $(\geq 180)(18)$, demonstrated shorter OS for patients with elevated pretreatment inflammatory blood markers (Figure S1).

The mechanisms underlying the association of high PLR and NLR and worse clinical outcomes in patients with cancer are unclear. Neutrophils can suppress anti- 


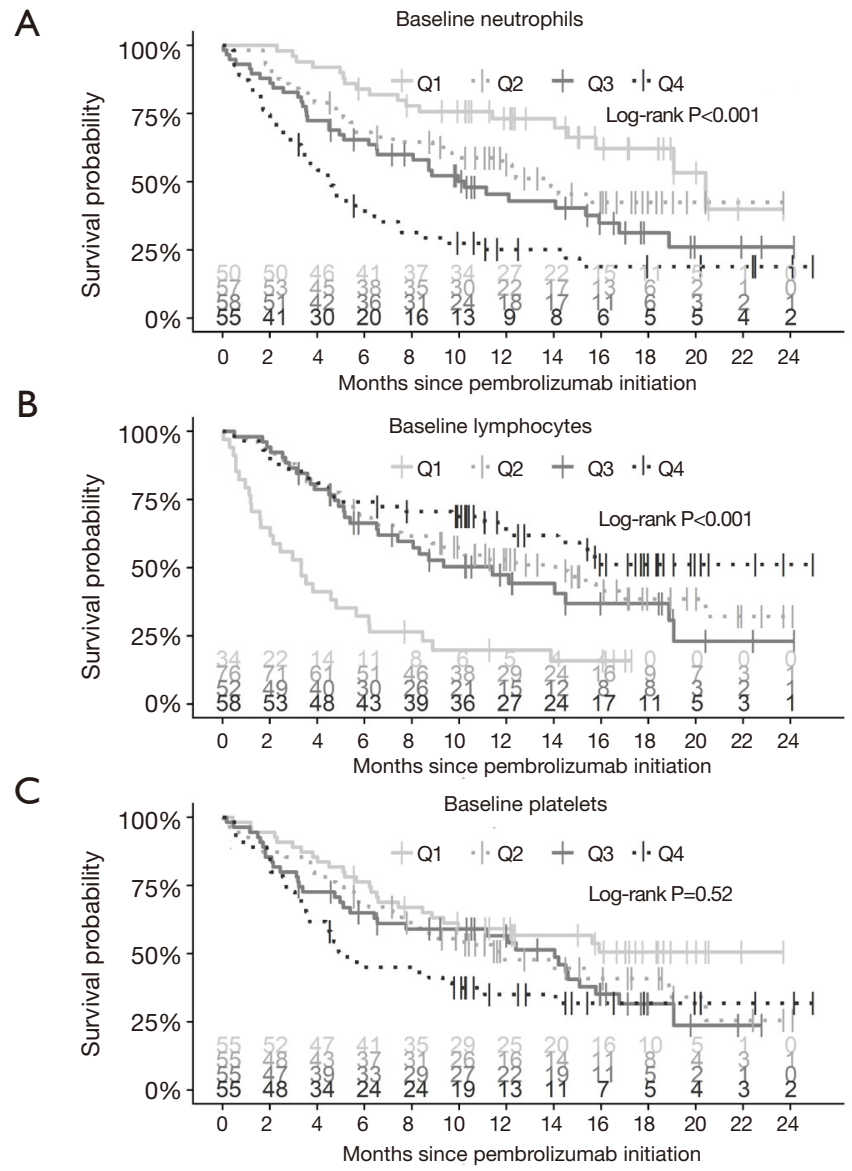

Figure 2 Kaplan-Meier curves of OS stratified by quartiles (Q) of baseline neutrophils (A), lymphocytes (B), and platelets (C). OS, overall survival.

tumor $\mathrm{T}$ cell responses and release pro-angiogenic factors that facilitate tumor cell dissemination (38). Platelets can similarly exert pro-tumor activities through elaboration of growth factors that stimulate cancer cell proliferation and facilitate the formation of metastatic foci (39). Conversely it is thought that lymphopenia represents impaired cellmediated immunity (40). As such the relative proportion of peripheral blood neutrophils (or platelets) to lymphocytes could potentially reflect a balance between protumor and antitumor immune phenotypes (5).

In the current series of patients treated in a real world setting, the incidence of any grade irAE (40.5\%), irAE $\geq$ grade $3(12.3 \%)$, and treatment discontinuation due to immune-toxicity (16.4\%) were generally higher than those reported in clinical trials (2-4). Identification of baseline clinical risk factors for irAE would facilitate patient counselling regarding treatment toxicities, improve safety monitoring protocols, and provide insights into irAE pathogenesis (24). Risk factors for irAE amongst pembrolizumab treated aNSCLC patients have included: history of autoimmune disease (20), female gender (41), elevated BMI (42), pre-existing auto-antibodies $(43,44)$, baseline derived NLR >3 (42), and baseline PLR $<180$ (23).

Poor baseline ECOG PS was the only studied risk factor in the current analysis associated with the development of irAE; specifically, the odds of developing immune toxicity were 3.76-fold higher at the 8-month landmark for patients with ECOG PS $2 / 3$ compared to ECOG PS 0/1. Importantly many of the previously mentioned publications on irAE risk factors excluded poor ECOG PS patients or did not explicitly state this patient characteristic. As immunotherapy can cause treatment related adverse events beyond irAE (such as fatigue) which impact quality of life, large prospective studies are required to clarify the safety 
Table 4 Distribution of irAE

\begin{tabular}{lcc}
\hline irAE & Any grade, $\mathrm{n}(\%)$ & Grade $\geq 3, \mathrm{n}(\%)$ \\
\hline All & $89(40.5)$ & $27(12.3)$ \\
Thyroid changes & $23(10.5)$ & $0(0.0)$ \\
Colitis & $21(9.5)$ & $4(1.8)$ \\
Dermatitis & $20(9.1)$ & $2(0.9)$ \\
Pneumonitis & $19(8.6)$ & $7(3.2)$ \\
Arthralgia & $13(5.9)$ & $1(0.5)$ \\
Hepatitis & $11(5.0)$ & $7(3.2)$ \\
Nephritis & $3(1.4)$ & $1(0.5)$ \\
Infusion reaction & $2(0.9)$ & $0(0.0)$ \\
Myositis & $2(0.9)$ & $2(0.9)$ \\
Neurologic & $2(0.9)$ & $0(0.0)$ \\
Pericarditis & $2(0.9)$ & $2(0.9)$ \\
Hematologic & $1(0.5)$ & $1(0.5)$ \\
Myasthenia gravis & $1(0.5)$ & $1(0.5)$ \\
Myocarditis & $1(0.5)$ & $1(0.5)$ \\
Myopathy & $1(0.5)$ & $1(0.5)$ \\
Pancreatitis & $1(0.5)$ & $0(0.0)$ \\
Polymyalgia rheumatic & $1(0.5)$ & $0(0.0)$ \\
\hline
\end{tabular}

irAE, immune-related adverse events; $n$, number of patients with irAE; \%, number of patients who developed irAE/220, thyroid changes include hyperthyroidism and hypothyroidism.

of pembrolizumab in this medically fragile population. It is also noteworthy that $30.9 \%$ of patients in the current series were $\geq 75$ years of age at diagnosis of aNSCLC and advanced age was not found to be a risk factor for irAE. This builds on a growing body of literature from real world studies regarding the safety of pembrolizumab in older aNSCLC patients (45).

We feel this study has important clinical relevance. The baseline characteristics of the cohort (including $40.5 \%$ of patients with ECOG PS 2/3) are consistent with a typical outpatient lung cancer clinic (22). In the era of immunotherapy, traditional prognostic factors derived from chemotherapy treated aNSCLC patients might not be applicable. NLR and PLR are easy to calculate and obtainable from standard of care tests. While the results of the current study require validation, knowledge of pre- treatment NLR and PLR ratios could provide useful prognostic information to counsel patients and their family members. ECOG PS was also significantly associated with shorter OS on multivariable analysis; however, determination of ECOG PS can be subjective and limited by intra- and interobserver variability (46).

Future research will be required to explore the predictive utility of NLR and PLR. Frontline treatment with platinum-based chemotherapy and pembrolizumab (vs. chemotherapy alone) improves OS for aNSCLC patients without EGFR/ALK alteration $(47,48)$. While an effective therapy, pembro-chemotherapy is resource intensive and can be associated with toxicity. Tumor mutation burden has been extensively studied as a potential predictive biomarker for efficacy of pembro-chemotherapy combination, although, no association has yet been found (49). Identification of a readily available biomarker such as NLR or PLR to determine which aNSCLC patients with PD-L1 TPS $\geq 50 \%$ would benefit from pembrolizumab monotherapy is an urgent unmet need. However, the current retrospective study cannot address this question as all patients received immunotherapy.

The present analysis has some limitations. First, the study was conducted in a retrospective manner and subject to selection bias and confounders. However, the studied population was similar in terms of receipt of pembrolizumab, PD-L1 TPS $\geq 50 \%$, and frontline treatment (95.5\% of cohort). Second, we did not examine for medications (such as steroids) at start of pembrolizumab therapy which could have affected NLR or PLR ratios. Last, diagnosis of irAE was based on judgement of the treating healthcare practitioner and not independently verified. Recent studies have highlighted discordance amongst clinicians in the diagnosis of irAE, especially in patients with multiple comorbidities (50).

\section{Conclusions}

In the current retrospective analysis of aNSCLC patients with PD-L1 TPS $\geq 50 \%$ receiving pembrolizumab as part of routine clinical care, high NLR and PLR were associated with shorter OS. Patients with ECOG PS 2/3 (compared to $0 / 1$ ) were observed to experience lower OS and higher odds for developing irAE. Given that NLR and PLR values are easily obtainable, prospective studies are warranted to confirm their prognostic significance and explore a 
predictive utility.

\section{Acknowledgments}

Funding: This work was supported by an unrestricted educational grant from AstraZeneca.

\section{Footnote}

Reporting Checklist: The authors have completed the REMARK reporting checklist. Available at http://dx.doi. org/10.21037/tlcr-20-541

Peer Review File: Available at http://dx.doi.org/10.21037/tlcr20-541

Conflicts of Interest: All authors have completed the ICMJE uniform disclosure form (available at http://dx.doi. org/10.21037/tlcr-20-541). Dr. DK has collected honoraria for continuing medical education events from Bristol Myers Squibb and Merck. Dr. AC has collected honoraria for continuing medical education events from Bristol Myers Squibb, Merck, AstraZeneca, Novartis, Pfizer, and Roche, and Takeda. Dr. ATF has collected honoraria for continuing medical education events from Bristol Myers Squibb. Dr. ZP has collected honoraria for continuing medical education events from Bristol Myers Squibb Canada, Merck, and AstraZeneca. The other authors have no conflicts of interest to declare.

Ethical Statement: The authors are accountable for all aspects of the work in ensuring that questions related to the accuracy or integrity of any part of the work are appropriately investigated and resolved. The study was conducted in accordance with the Declaration of Helsinki (as revised in 2013). The study was approved by the institutional ethics board of University of British Columbia Research Ethics Board (H19-01328) and individual consent for this retrospective analysis was waived.

Open Access Statement: This is an Open Access article distributed in accordance with the Creative Commons Attribution-NonCommercial-NoDerivs 4.0 International License (CC BY-NC-ND 4.0), which permits the noncommercial replication and distribution of the article with the strict proviso that no changes or edits are made and the original work is properly cited (including links to both the formal publication through the relevant DOI and the license). See: https://creativecommons.org/licenses/by-nc-nd/4.0/.

\section{References}

1. Garon EB, Rizvi NA, Hui R, et al. Pembrolizumab for the treatment of non-small-cell lung cancer. N Engl J Med 2015;372:2018-28.

2. Herbst RS, Baas P, Kim D, et al. Pembrolizumab versus docetaxel for previously treated, PD-L1-positive, advanced non-small-cell lung cancer (KEYNOTE-010): a randomized controlled trial. Lancet 2016;387:1540-50.

3. Reck M, Rodriguez-Abreu D, Robinson AG, et al. Updated analysis of KEYNOTE-024: pembrolizumab versus platinum-based chemotherapy for advanced nonsmall-cell lung cancer with PD-L1 tumor proportion score of 50\% or greater. J Clin Oncol 2019;37:537-46.

4. Mok TSK, Wu YL, Kudaba I, et al. Pembrolizumab versus chemotherapy for previously untreated, PD-L1expressing, locally advanced or metastatic non-small-cell lung cancer (KEYNOTE-042): a randomised, open-label, controlled, phase 3 trial. Lancet 2019;393:1819-30.

5. Park W, Lopes G. Perspectives: neutrophil-to-lymphocyte ratio as a potential biomarker in immune checkpoint inhibitor for non-small-cell lung cancer. Clin Lung Cancer 2019;20:143-7.

6. Rizvi H, Sanchez-Vega F, La K, et al. Molecular determinants of response to anti-programmed cell death (PD)-1 and anti-programmed death-ligand 1 (PD-L1) blockade in patients with non-small-cell lung cancer profiled with targeted next-generation sequencing. J Clin Oncol 2018;36:633-41.

7. Diakos CI, Charles KA, McMillan DC, et al. Cancerrelated inflammation and treatment effectiveness. Lancet Oncol 2014;15:e493-503.

8. Templeton AJ, McNamara MG, Šeruga B, et al. Prognostic role of neutrophil-to-lymphocyte ratio in solid tumors: a systematic review and meta-analysis. J Natl Cancer Inst 2014;106:dju124.

9. Templeton AJ, Ace O, McNamara MG, et al. Prognostic role of platelet to lymphocyte ratio in solid tumors: a systematic review and meta-analysis. Cancer Epidemiol Biomarkers Prev 2014;23:1204-12.

10. Bagley SJ, Kothari S, Aggarwal C, et al. Pretreatment neutrophil-to-lymphocyte ratio as a marker of outcomes in nivolumab-treated patients with advanced non-small-cell lung cancer. Lung Cancer 2017;106:1-7. 
11. Diem S, Schmid S, Krapf M, et al. Neutrophil-toLymphocyte ratio (NLR) and Platelet-to-Lymphocyte ratio (PLR) as prognostic markers in patients with nonsmall cell lung cancer (NSCLC) treated with nivolumab. Lung Cancer 2017;111:176-81.

12. Zer A, Sung MR, Walia P, et al. Correlation of neutrophil to lymphocyte ratio and absolute neutrophil count with outcomes with PD-1 axis inhibitors in patients with advanced non-small-cell lung cancer. Clin Lung Cancer 2018;19:426-34.e1.

13. Soyano AE, Dholaria B, Marin-Acevedo JA, et al. Peripheral blood biomarkers correlate with outcomes in advanced non-small cell lung Cancer patients treated with anti-PD-1 antibodies. J Immunother Cancer 2018;6:129.

14. Patil PD, Khunger M, Rakshit S, et al. Pre-treatment hematological markers as a predictive biomarker for survival in patients with non-small cell lung cancer treated with nivolumab. J Clin Oncol 2017;15:11547.

15. Nakaya A, Kurata T, Yoshioka H, et al. Neutrophilto-lymphocyte ratio as an early marker of outcomes in patients with advanced non-small-cell lung cancer treated with nivolumab. Int J Clin Oncol 2018;23:634-40.

16. Suh KJ, Kim SH, Kim YJ, et al. Post-treatment neutrophil-to-lymphocyte ratio at week 6 is prognostic in patients with advanced non-small cell lung cancers treated with anti-PD-1 antibody. Cancer Immunol Immunother 2018;67:459-70.

17. Qi Y, Liao D, Fu X, et al. Elevated platelet-to-lymphocyte corresponds with poor outcome in patients with advanced cancer receiving anti-PD-1 therapy. Int Immunopharmacol 2019;74:105707.

18. Gu X, Sun S, Gao XS, et al. Prognostic value of platelet to lymphocyte ratio in non-small cell lung cancer: evidence from 3,430 patients. Sci Rep 2016;6:23893.

19. Mezquita L, Auclin E, Ferrara R, et al. Association of the lung immune prognostic index with immune checkpoint inhibitor outcomes in patients with advanced non-small cell lung cancer. JAMA Oncol 2018;4:351-7.

20. Champiat S, Lambotte O, Barreau E, et al. Management of immune checkpoint blockade dysimmune toxicities: a collaborative position paper. Ann Oncol 2016;27:559-74.

21. Hutchins LF, Unger JM, Crowley JJ, et al. Underrepresentation of patients 65 years of age or older in cancer-treatment trials. N Engl J Med 1999;341:2061-7.

22. Lilenbaum RC, Cashy J, Hensing TA, et al. Prevalence of poor performance status in lung cancer patients: implications for research. J Thorac Oncol 2008;3:125-9.

23. Pavan A, Calvetti L, Dal Maso A, et al. Peripheral blood markers identify risk of immune-related toxicity in advanced non-small cell lung cancer treated with immunecheckpoint inhibitors. Oncologist 2019;24:1128-36.

24. Johnson DB, Balko JM. Biomarkers for immunotherapy toxicity: are cytokines the answer? Clin Cancer Res 2019;25:1452-54.

25. McShane LM, Altman DG, Sauerbrei W, et al. REporting recommendations for tumour MARKer prognostic studies (REMARK). Br J Cancer 2005;93:387-91.

26. BC Cancer Protocol Summary for Treatment of Advanced Non-Small Cell Lung Cancer Using Pembrolizumab. Available online: http://www.bccancer. bc.ca/chemotherapy-protocols-site/Documents/Lung/ ULUAVPMB_Protocol.pdf(Accessed 15 May 2020).

27. Agilent. PD-L1 IHC 22C3 pharmDx Advancing Immunotherapy Options. Available online: https:// www.agilent.com/en/product/pharmdx/pd-11-ihc-22c3pharmdx (Accessed 15 May 2020).

28. U.S. Department of Health and Human Services. Common terminology criteria for adverse events (CTCAE) Version 4.0. 2009. Available online: https:// evs.nci.nih.gov/ftp1/CTCAE/CTCAE_4.03/Archive/ CTCAE_4.0_2009-05-29_QuickReference_8.5x11.pdf (Accessed 26 February 2020).

29. Schemper M, Smith TL. A note on quantifying followup in studies of failure time. Control Clin Trials 1996;17:343-6.

30. Delgado J, Pereira A, Villamor N, et al. Survival analysis in hematologic malignancies: recommendations for clinicians. Haematologica 2014;99:1410-20.

31. R Core Team. R: A language and environment for statistical computing. R Foundation for Statistical Computing, Vienna, Austria. 2019. Available online: https://www.R-project.org/

32. Therneau TM, Grambsch PM. Modeling Survival Data: Extending the Cox Model. New York: Springer, 2000.

33. Therneau T. A Package for Survival Analysis in S. R package version 2.44. 2019. Available online: https:// CRAN.R-project.org/package=survival

34. Torsten Hothorn. maxstat: Maximally Selected Rank Statistics. R package version 0.7-25. 2017. Available online: https://CRAN.R-project.org/package=maxstat

35. Kassambara A, Kosinski M, Biecek P. survminer: drawing survival curves using 'ggplot2'. R package version 0.4.6. 2019. Available online: https:/CRAN.R-project.org/ package=survminer (Accessed 26 February 2020).

36. Yoshida K. tableone: Create 'Table 1' to Describe Baseline Characteristics. R package version 0.10.0. 2019. Available 
online: https://CRAN.R-project.org/package=tableone

37. Hasegawa T, Yanagitani N, Utsumi H, et al. Association of high neutrophil-to-lymphocyte ratio with poor outcomes of pembrolizumab therapy in high-PD-L1-expressing nonsmall cell lung cancer. Anticancer Res 2019;39:6851-7.

38. Swierczak A, Mouchemore KA, Hamilton JA, et al. Neutrophils: important contributors to tumor progression and metastasis. Cancer Metastasis Rev 2015;34:735-51.

39. Schlesinger M. Role of platelets and platelet receptors in cancer metastasis. J Hematol Oncol 2018;11:125.

40. Grivennikov SI, Greten FR, Karin M. Immunity, inflammation, and cancer. Cell 2010;140:883-99.

41. Duma N, Abdel-Ghani A, Yadav S, et al. Sex differences in tolerability to anti-programmed cell death protein 1 therapy in patients with metastatic melanoma and nonsmall cell lung cancer: are we all equal? Oncologist 2019;24:e1148-55.

42. Eun Y, Kim IY, Sun JM, et al. Risk factors for immunerelated adverse events associated with anti-PD-1 pembrolizumab. Sci Rep 2019;9:14039.

43. Toi Y, Sugawara S, Sugisaka J, et al. Profiling preexisting antibodies in patients treated with anti-PD-1 therapy for advanced non-small cell lung cancer. JAMA Oncol 2019;5:376-83.

44. Osorio JC, Ni A, Chaft JE, et al. Antibody-mediated thyroid dysfunction during T-cell checkpoint blockade

Cite this article as: Ksienski D, Wai ES, Alex D, Croteau NS, Freeman AT, Chan A, Patterson T, Clarkson M, Fiorino L, Poonja Z, Fenton D, Irons S, Lesperance M. Prognostic significance of the neutrophil-to-lymphocyte ratio and platelet-to-lymphocyte ratio for advanced non-small cell lung cancer patients with high PD-L1 tumor expression receiving pembrolizumab. Transl Lung Cancer Res 2021;10(1):355-367. doi: $10.21037 /$ tlcr-20-541 in patients with non-small-cell lung cancer. Ann Oncol 2017;28:583-9.

45. Ksienski D, Wai ES, Croteau NS, et al. Association of age with differences in immune related adverse events and survival of patients with advanced nonsmall cell lung cancer receiving pembrolizumab or nivolumab. J Geriatr Oncol 2020;11:807-13.

46. Ando M, Ando Y, Hasegawa Y, et al. Prognostic value of performance status assessed by patients themselves, nurses, and oncologists in advanced non-small cell lung cancer. $\mathrm{Br}$ J Cancer 2001;85:1634-9.

47. Gandhi L, Rodriguez-Abreu D, Gadgeel S, et al. Pembrolizumab plus chemotherapy in metastatic nonsmall-cell lung cancer. N Engl J Med 2018;378:2078-92.

48. Paz-Ares L, Luft A, Vicente D, et al. Pembrolizumab plus chemotherapy for squamous non-small-cell lung cancer. $\mathrm{N}$ Engl J Med 2018;379:2040-51.

49. Pas-Ares L, Langer CJ, Novello S, et al. Pembrolizumab plus platinum-based chemotherapy for metastatic NSCLC: tissue TMB (tTMB) and outcomes in KEYNOTE-021, 189, and 407. Barcelona: Lecture presented at European Society for Medical Oncology (ESMO) Congress, 2019.

50. Hsiehchen D, Watters MK, Lu R, et al. Variation in the assessment of immune-related adverse event occurrence, grade, and timing in patients receiving immune checkpoint inhibitors. JAMA Netw Open 2019;2:e1911519. 


\section{Supplementary}
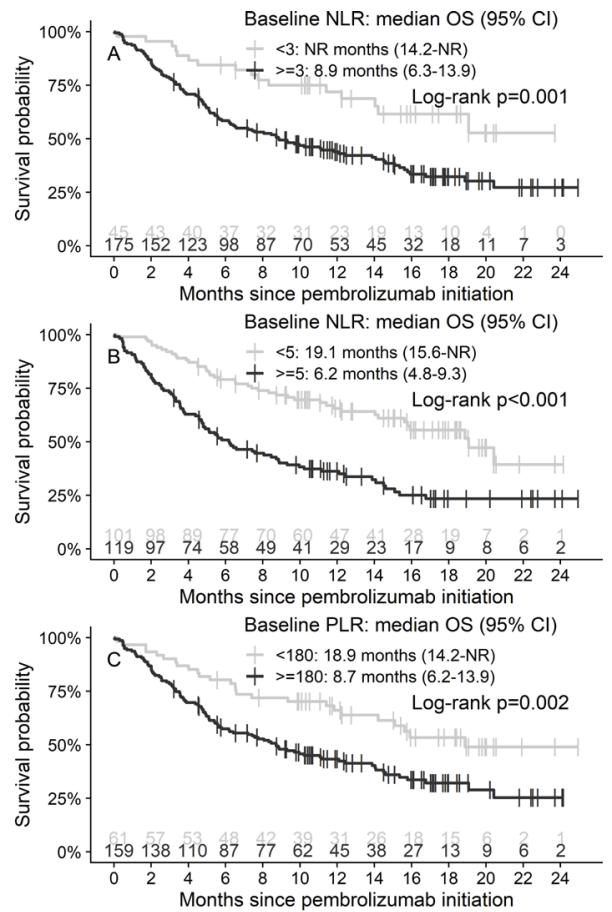

Figure S1 Kaplan-Meier curves of OS according to baseline (A) NLR ( $\geq 3 v s .<3$ ), (B) NLR $\geq 5$ vs. $<5$, and (C) PLR ( $\geq 180 v s$. $<180$ ). OS, overall survival; NLR, neutrophil-to-lymphocyte ratio; PLR, platelet-to-lymphocyte ratio; CI, confidence interval; NR, not reached. 
Table S1 Logistic regression of irAE development within 6 months of pembrolizumab initiation

\begin{tabular}{|c|c|c|c|c|}
\hline Variables & \multicolumn{2}{|c|}{ Univariable } & \multicolumn{2}{|c|}{ Multivariable } \\
\hline Age ( $\geq 75$ vs. $<75)$ & $0.52(0.25-1.05)$ & 0.07 & $0.57(0.26-1.22)$ & 0.15 \\
\hline Gender (male vs. female) & $1.30(0.68-2.51)$ & 0.43 & $1.20(0.59-2.43)$ & 0.62 \\
\hline ECOG PS (2/3 vs. 0/1) & $1.98(1.00-3.95)$ & 0.051 & $1.88(0.92-3.92)$ & 0.09 \\
\hline Brain metastases (present vs. none) & $0.64(0.21-1.78)$ & 0.40 & $0.52(0.15-1.64)$ & 0.28 \\
\hline Histology (squamous vs. non-squamous) & $1.30(0.59-2.87)$ & 0.51 & $0.90(0.38-2.12)$ & 0.82 \\
\hline Baseline NLR ( $\geq 6.4$ vs. <6.4) & $1.29(0.65-2.56)$ & 0.46 & $0.85(0.36-1.94)$ & 0.70 \\
\hline Baseline PLR ( $\geq 441.8$ vs. $<441.8$ ) & $2.09(0.88-5.16)$ & 0.10 & $2.14(0.75-6.40)$ & 0.16 \\
\hline
\end{tabular}

irAE, immune-related adverse events; OR, odds ratio; Cl, confidence interval; ECOG PS, Eastern Cooperative Oncology Group performance status; NLR, neutrophil-to-lymphocyte ratio; PLR, platelet-to-lymphocyte ratio.

Table S2 Logistic regression of irAE development within 8 months of pembrolizumab initiation

\begin{tabular}{|c|c|c|c|c|}
\hline Variables & \multicolumn{2}{|c|}{ Univariable } & \multicolumn{2}{|c|}{ Multivariable } \\
\hline Age ( $\geq 75$ vs. $<75)$ & $0.62(0.30-1.27)$ & 0.19 & $0.66(0.29-1.47)$ & 0.31 \\
\hline Gender (male vs. female) & $1.12(0.57-2.21)$ & 0.74 & $1.02(0.48-2.18)$ & 0.95 \\
\hline ECOG PS (2/3 vs. 0/1) & $3.46(1.62-7.75)$ & 0.002 & $3.76(1.69-8.88)$ & 0.002 \\
\hline Brain metastases (present vs. none) & $0.70(0.25-1.96)$ & 0.50 & $0.44(0.12-1.44)$ & 0.18 \\
\hline Histology (squamous vs. non-squamous) & $1.02(0.45-2.36)$ & 0.96 & $0.59(0.22-1.53)$ & 0.28 \\
\hline Baseline NLR ( $\geq 6.4$ vs. $<6.4)$ & $1.27(0.62-2.63)$ & 0.51 & $0.79(0.32-1.94)$ & 0.62 \\
\hline Baseline PLR ( $\geq 441.8$ vs. $<441.8$ ) & $2.13(0.84-5.90)$ & 0.12 & $2.68(0.85-9.29)$ & 0.10 \\
\hline
\end{tabular}

irAE, immune-related adverse events; OR, odds ratio; Cl, confidence interval; ECOG PS, Eastern Cooperative Oncology Group performance status; NLR, neutrophil-to-lymphocyte ratio; PLR, platelet-to-lymphocyte ratio. 